\title{
Factores determinantes en la adaptación del paciente portador de ostomía y la importancia del estomaterapeuta
}

\author{
Miriam Lizeth Cantero Cunalata \\ al226396@uji.es \\ ELADIO JOAQUín COLLAdO BoIRA \\ colladoe@uji.es
}

\section{Resumen}

Introducción: Las personas enterostomizadas suelen experimentar problemas fisiológicos, psicológicos y sociales que repercuten en su adaptación social. El objetivo de este trabajo es identificar las principales variables que intervienen en la adaptación de los pacientes a la enterostomía, así como dar a conocer la importancia de la labor del estomaterapeuta en este proceso. Método: Búsqueda bibliográfica de revisiones sistemáticas relacionadas con el objeto de estudio, indexadas en scopus, PubMed y ClNAHL. La recuperación de información se llevó a cabo mediante la combinación de vocabulario estructurado y texto libre, y el uso de operadores booleanos "AND» y "OR» para delimitar la búsqueda. Se seleccionaron todas aquellas evidencias científicas que cumplen con los criterios de elegibilidad establecidos. La calidad metodológica de los estudios se evaluó mediante una herramienta digital. Resultados: Se identificaron 1238 referencias, incluyéndose finalmente dos revisiones sistemáticas que cumplían los criterios de elegibilidad establecidos. Los artículos sintetizan los resultados de 29 estudios cualitativos y cuantitativos. Conclusiones: La educación sanitaria, la alteración de la imagen corporal, el apoyo familiar, la comunicación del diagnóstico de cáncer y el proceso de autocuidado repercuten principalmente en la adaptación del paciente. Son necesarias nuevas investigaciones para conseguir conclusiones fiables y centrarlas en el papel del estomaterapeuta. Se recomienda garantizar el acceso a los pacientes ostomizados a una atención especializada e incluir su cuidado en los programas formativos.

Palabras clave: ostomía, colostomía, cuidados de enfermería, rol de la enfermera, adaptación.

\section{Abstract}

Introduction: The ostomates often experience physiological, psychological and social problems that affect their social adaptation. The aims of this study are to identify the main variables involved in the adaptation of patients to enterostomy as well as raising awareness of the importance of the work of the ostomy specialist in this process. Method: Literature research of systematic reviews related to the object of study, indexed 
in SCOPUS, PubMed and CINAHL. The information retrieval was performed by combining structured vocabulary and free text, and the use of Boolean operators "AND» and «OR» to narrow the search. All the scientific evidence that meets the eligibility criteria was selected. The methodological quality of the studies was assessed using a digital tool. Results: 1238 references were identified, eventually including two systematic reviews that met the eligibility criteria. Articles summarize 29 qualitative and quantitative studies results. Conclusions: Health education, altered body image, family support, cancer diagnosis communication and the self-care process particularly affect patient's adaptation. Additional research is needed to get reliable conclusions, focusing on the ostomy specialist's role. It is highly recommended ensuring access to specialized care to ostomates and including their care on training programs.

Keywords: ostomy, colostomy, nursing care, nurse's role, adaptation.

\section{Introducción}

Una ostomía es el resultado de un procedimiento quirúrgico con fines terapéuticos que comunica o fistuliza una víscera hueca con la superficie corporal. La mucosa se sutura a la piel del abdomen en una zona distinta al orificio natural de evacuación, denominándose estoma, término que etimológicamente procede del griego y cuyo significado es 'boca' o 'apertura'. Así pues, existen diferentes tipos de estoma en función de la porción intestinal seccionada y de la temporalidad de su permanencia.

Según la zona intestinal exteriorizada puede denominarse colostomía cuando se comunica el colon con la pared abdominal, mientras que se designa como ileostomía cuando la parte que aboca a la pared abdominal es una porción de íleon. Asimismo, en función del tiempo de permanencia, el estoma puede ser temporal o definitivo, dependiendo de la patología o lesión intestinal que produjo la realización de la intervención, es decir, depende de la posibilidad de recuperación de la función intestinal una vez haya cumplido con su cometido. (Aecc, 2014; Canaval, Londoño y Herrera, 2005; Instituto Antae, 2014).

En el presente trabajo nos centraremos en los estomas intestinales de eliminación, también denominados enterostomías de eliminación.

Los principales factores etiológicos que conllevan a la realización de una enterostomía de eliminación son el cáncer colorrectal (CCR), el cual representa un $80 \%$ de las enterostomías que se realizan en España, seguida de la enfermedad Inflamatoria Intestinal (colitis ulcerosa y enfermedad de Crohn), que representa un $8 \%$ del total de las enterostomías efectuadas, entre otras como la diverticulitis intestinal, los traumatismos anorrectales o la oclusión intestinal, las cuales representan un menor porcentaje (Campillo, 2014; Collado, 2014).

Las enterostomías de eliminación conllevan la adquisición de competencias para el paciente ostomizado o su cuidador principal, relacionados con el cuidado e higiene del orificio estomal, la utilización de dispositivos recolectores específicos, el aprendizaje de técnicas de continencia, así como la adopción de unos hábitos alimenticios adecuados a fin de evitar molestias relacionadas con los gases intestinales, la consistencia de las heces y el olor de las mismas.

Si bien las funciones fisiológicas del organismo continúan funcionando igual que antes de la intervención, la presencia del estoma en la pared abdominal produce un gran impacto a nivel emocional, psicológico, físico y social en este tipo de pacientes, sobre todo por el desconoci- 
miento de este tipo de cirugía, sus consecuencias y la ausencia de información presente en algunas ocasiones (Instituto Antae, 2014).

A pesar de la ausencia de un registro nacional de pacientes ostomizados que nos permita conocer con exactitud la cantidad de pacientes portadores de ostomía, según datos de la Asociación Española de Cirujanos (2005) y de algunos estudios recientes (Collado, 2014, Instituto Antae, 2014), en países occidentales se recogen cifras que se encuentran entre el 2 y el 4 por mil de personas adultas ostomizadas, mientras que entre la sociedad española es aproximadamente el $1,5 \%$, lo que supone un colectivo de unas 70.000 personas, siendo la incidencia en la población adulta de más de un $3 \%$, produciéndose cada año más de 13.000 casos nuevos, cifras que justifican el fuerte impacto social y sanitario del problema en cuestión.

Según estudios recientes (Collado, 2014; Instituto Antae, 2014), entre el $16 \%$ y el $20 \%$ de los pacientes enterostomizados presenta algún tipo de complicación, entre las que destacan por su frecuencia y repercusión en la calidad de vida del paciente la dermatitis, edema, necrosis, hemorragia, restracción, prolapso, evisceración y diarrea (Collado, 2014; Corella y cols., 2005; Grupo CIDO, 1997).

Se evidencia, por tanto, la importancia de la educación del autocuidado tan pronto como sea posible, ya sea tanto para evitar como para minimizar estos problemas, en el que el personal de enfermería especializado juega un papel fundamental (Collado, 2014; Instituto Antae, 2014).

Dado que la repercusión de este tipo de intervención sobre la calidad de vida de los pacientes enterostomizados ha quedado demostrada en múltiples estudios (Brown y Randle, 2005; Collado, 2014; Instituto Antae, 2014), es de relevancia el beneficio de la participación de un equipo multidisciplinar, que dirija el cuidado de la enterostomía hacia la independencia de los usuarios y, de esta forma, éstos puedan dirigir sus potencialidades hacia formas que les ayuden a afrontar su nueva condición de vida (Mejía, 2006). Este cuidado es realizado sobre todo por el/la enfermero/a especializado/a o estomaterapeuta, que es el profesional que garantiza esta atención adecuada tanto al paciente ostomizado como a su familia, ya que le proporciona una educación sanitaria que le capacita para el cuidado de su estoma. Además, previene, detecta y trata complicaciones, y le presta el apoyo emocional que precisa para afrontar de manera óptima este proceso (Instituto Antae, 2014).

La enfermería, por tanto, tiene un papel clave en el cuidado de este tipo de usuarios durante el perioperatorio, realizando labores de ayuda al paciente a entender mejor su patología, facilitando la adaptación a la vida con un estoma, enseñando habilidades prácticas para el cuidado del mismo, así como abordando temas relacionados con la imagen corporal, sus redes de apoyo familiar, el empleo, y la sexualidad, es decir, el personal enfermero se dispone a enseñar, supervisar y acompañar al individuo durante todo el proceso de adaptación a su vida cotidiana (Brown y Randle, 2005).

A pesar del impacto en la calidad de vida del paciente y de la necesidad de aprendizaje de cuidados tanto para evitar complicaciones como para reducir costes, el $40 \%$ de los hospitales públicos de España no cuenta con una consulta de ostomías. Asimismo, de los 220 hospitales públicos solo hay consultas en 146, siendo además el $73 \%$ de las consultas de ostomías a tiempo parcial y solo el $27 \%$ a tiempo completo. Por tanto, el $36 \%$ de los ostomizados procedentes de hospitales públicos no tiene acceso a una atención especializada en ostomías, lo que se traduce en un aumento de las complicaciones y, por consiguiente, de los costes sanitarios, así como la afectación de la acomodación del usuario a su nuevo estilo de vida, es decir, se produce una repercusión negativa en la calidad de vida tanto del individuo portador del estoma como de su familia (Instituto Antae, 2014). Por ello, el propósito de este trabajo de revisión bibliográfica es identificar los principales factores que afectan a la adaptación del paciente a su enterostomía 
y a su readaptación a su vida diaria, así como mostrar la importancia de la figura del estomaterapeuta como ayuda en dicha adaptación.

\section{Método}

Se ha realizado una revisión bibliográfica con el propósito de recuperar las mejores evidencias científicas en el ámbito de la disciplina enfermera. Definiendo para ello como criterios de inclusión: revisiones sistemáticas publicadas en los últimos cinco años, escritas en inglés y castellano, que tratan sobre la adaptación del paciente a su ostomía y el papel de enfermería dentro de esta situación. Únicamente se eligieron revisiones sistemáticas debido a que éstas resumen los resultados de los estudios disponibles y proporcionan un alto nivel de evidencia sobre la eficacia de las intervenciones en temas de salud (Centro Cochrane Iberoamericano, 2014).

La búsqueda bibliográfica se llevó a cabo entre los meses de enero a marzo de 2015 en las bases de datos SCOPUS, PUBMED y CINAHL, ya que se consideran unas de las fuentes de información más importantes en ciencias de la salud y son actualizadas constantemente, siendo además CINAHL una base de datos especializada en enfermería.

Con el fin de realizar una búsqueda eficiente de información, se formuló la pregunta clínica mediante el modelo PIO, que permite maximizar la recuperación de evidencias en las bases de datos, enfoca el propósito de la investigación y evita realizar búsquedas innecesarias (Costa, Mattos y Cuce, 2007). La pregunta fue estructurada en los siguientes componentes:

$$
\begin{aligned}
& \text { P - Ostomía/colostomía } \\
& \text { I - Cuidado de enfermería/papel de enfermería } \\
& \text { O - Adaptación }
\end{aligned}
$$

Para la realización de la búsqueda bibliográfica, estos elementos se utilizaron combinando vocabulario estructurado o descriptores y texto libre. Asimismo, para delimitar la búsqueda se utilizaron los operadores booleanos «AND» (operador restrictivo) y "OR» (operador de adición), así como los filtros disponibles en cada una de las bases de datos.

La estrategia de búsqueda definitiva fue: (ostomy OR colostomy) AND (nursing care OR nurse's role) AND (adjustment OR adaptation).

A continuación, se revisaron los títulos y resúmenes de los artículos resultantes de la búsqueda final, seleccionándose aquellas referencias que cumplían con los criterios de elegibilidad establecidos y se podía acceder al texto completo.

La evaluación de la calidad metodológica de las publicaciones incluidas, se realizó a través de la plataforma www.lecturacritica.com, mediante unos instrumentos metodológicos denominados "Fichas de lectura crítica», las cuales permiten valorar diferentes diseños de estudios, analizar la calidad o validez de éstos, facilitando la síntesis de la evidencia de manera clara y visible en tablas (López y cols., 2006).

\section{Resultados}

La búsqueda realizada en la base de datos scopus recuperó 532 referencias, de las cuales 1 cumplía con los criterios de elegibilidad mencionados anteriormente y ha sido incluida en esta revisión (Araujo y Neves, 2014). De la base de datos PUBMED se recuperaron 283 referencias, de las cuales 1 cumplía con los criterios de elegibilidad (Seng, Chen, Chiew, Hong-Gu y 
Klainin, 2013). Mientras que de la búsqueda realizada en la base de datos CINAHL se recuperaron 423 referencias, de las cuales 1 ha sido incluida en este trabajo al cumplir con los criterios de elegibilidad, siendo la misma referencia que la recuperada en la base de datos PUBMED. EI proceso de selección de los estudios se describe en la figura 1.

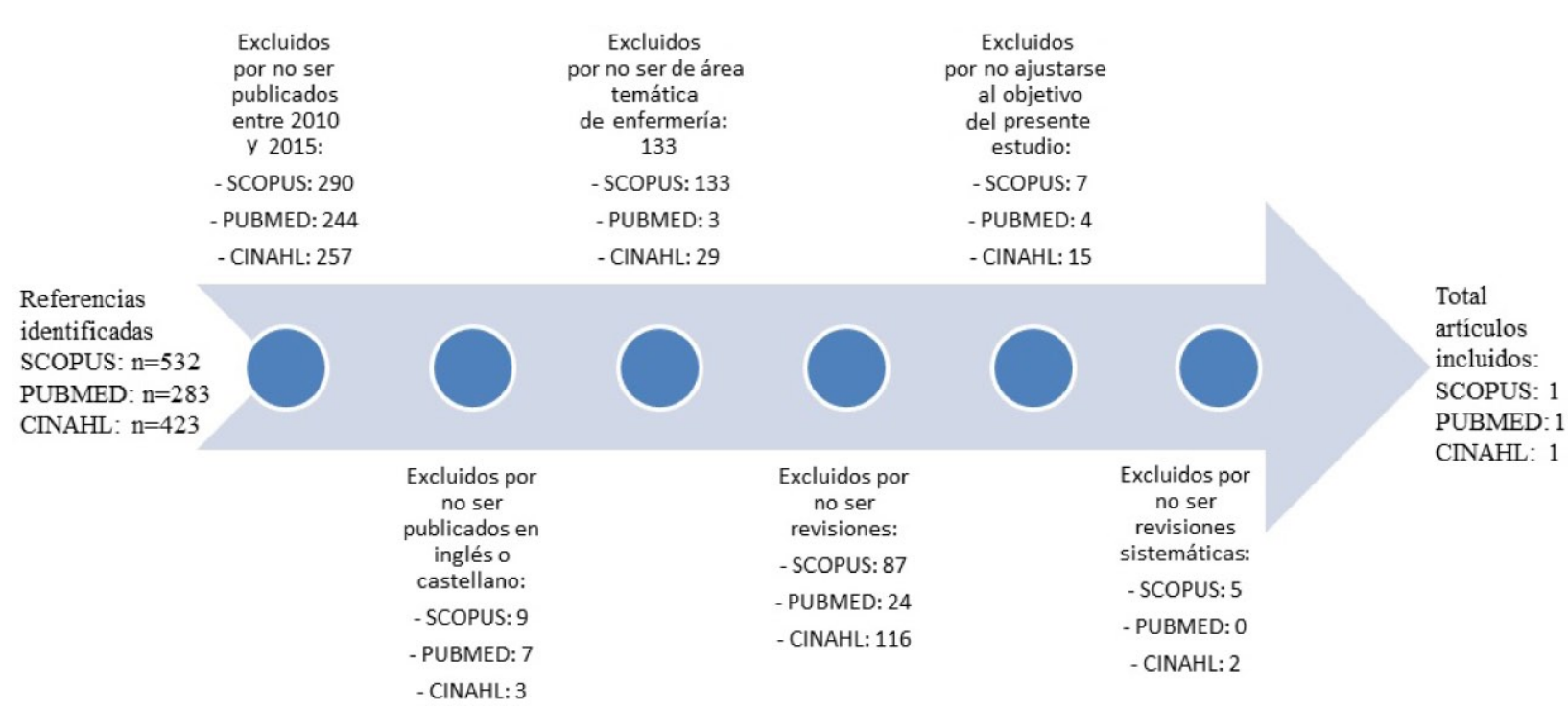

Figura 1. Proceso de selección de los estudios

Las dos revisiones sistemáticas incluidas en este trabajo (Araujo y Neves, 2014; Seng, Chen, Chiew, Hong-Gu y Klainin, 2013) sintetizan los resultados de 29 estudios cualitativos y cuantitativos.

La revisión sistemática realizada por Araujo y Neves (2014) incluye 21 artículos, de los cuales once son cualitativos y diez, cuantitativos. Los autores procedieron a realizar un análisis de contenido de las evidencias recuperadas, siendo clasificadas en base al análisis temático de Bardin (2002), que consiste en tres etapas: preanálisis, descripción analítica e interpretación inferencial. De este análisis surgieron las tres categorías desarrolladas en el estudio.

En este estudio no se tiene en cuenta el posible sesgo de publicación (Marín, Sánchez y López, 2009), ya que no describe el tamaño muestral de las evidencias recuperadas.

En la revisión sistemática de Seng y cols. (2013), se incluyen 8 artículos, de los cuales tres son de tipo cualitativo y cinco de diseño cuantitativo. Los estudios incluidos fueron evaluados utilizando las herramientas elaboradas por el Instituto Joanna Briggs (The Joanna Briggs Institute, 2011). Todas las evidencias científicas incluidas usaban un muestreo por conveniencia, lo que puede incrementar el riesgo de sesgo de selección y limitar la generalización de los resultados.

La adopción de un diseño transversal en 7 de los artículos no proporcionó pruebas sólidas para apoyar las relaciones causales entre las variables de estudio. Además, se observó una limitación al no especificar el período de búsqueda.

En esta revisión (Seng y cols., 2013), por una parte se tiene en cuenta el posible sesgo de publicación al reportar el tamaño muestral de los estudios incluidos. Sin embargo, al incluir en la revisión solo trabajos publicados en inglés, aumenta la posibilidad de sesgo de publica- 
ción, pudiendo producirse una sobrevaloración del efecto de las variables evaluadas, ya que se ha probado que los estudios con resultados no significativos o en idioma distinto al inglés tienen menor probabilidad de ser publicados en revistas de alto impacto o indexadas en las bases de datos de mayor relevancia, por lo que a su vez tienen menor posibilidad de ser incluidos en revisiones sistemáticas (Letelier, Manríquez y Rada, 2005; Pértega y Pita, 2005).

Los criterios de elegibilidad están claramente establecidos en ambos estudios, y las bases de datos empleadas son relevantes y con un peso importante en las ciencias de la salud, a pesar de no explicar los motivos de la elección de las mismas.

La evaluación del grado de heterogeneidad de los estudios incluidos en las revisiones no es realizada por los autores de ambas publicaciones. Asimismo, ambos estudios no declaran la existencia o ausencia de algún conflicto de interés, lo que puede aumentar el riesgo de sesgo de publicación.

\section{Discusión y conclusiones}

Tras el análisis de ambos artículos incluidos en esta revisión bibliográfica, se ha observado que los dos estudios refieren resultados similares, siendo una de las principales variables que repercuten en la adaptación del paciente a la enterostomía de eliminación, la educación aportada por el personal de enfermería acerca del cuidado del estoma.

Según Araujo y Neves (2014) se debería prestar una atención individualizada que tenga en cuenta las actitudes del paciente, la base de conocimientos y la capacidad de aprendizaje de éste y de su familia, con el objetivo de conseguir un autocuidado eficaz. Para ello, el personal de enfermería debería ampliar sus conocimientos y aumentar su comprensión de los sentimientos que surgen en este tipo de usuarios durante el proceso de adaptación a la ostomía. En esto coincide el estudio de Seng y cols. (2013) en el que expone que los/as enfermeros/as deberían ser conscientes de la importancia de cuidar tanto el bienestar físico como el psicológico de los pacientes. Además, Araujo y Neves (2014) proponen que el refuerzo de fundamentos teóricos y la actualización de conocimientos de los estudiantes y profesionales de enfermería son elementos fundamentales para el desarrollo de habilidades afectivas que les permitirán proporcionar una atención adecuada en la práctica clínica a este tipo de usuarios, lo que se asemeja a los resultados de publicaciones recientes (Gómez y cols., 2013).

Otra de las variables más importantes en las que coinciden ambos estudios y que pueden influir en la adaptación a la vida diaria del individuo, es la alteración de la imagen corporal debido a la presencia del estoma y a los distintos dispositivos que deben utilizar. Esto origina la aparición de sentimientos negativos que disminuyen su autoestima y que intervienen de forma adversa en su sexualidad y en el retorno a las actividades sociales, originando en muchas ocasiones problemas de aislamiento social y, por ende, repercute negativamente en su calidad de vida. Asimismo, ambos estudios refieren que la aceptación de la nueva imagen corporal es primordial para que el paciente acepte el estoma y se integre nuevamente en la sociedad, lo que también coincide con otros trabajos realizados recientemente (Bonill y cols., 2013; Cotrim y Pereira, 2008; Gómez y cols., 2013; Martín, Panduro, Crespillo, Rojas y González, 2010).

Del mismo modo, en ambas revisiones se tiene en cuenta el proceso de autocuidado como otra de las variables a considerar, ya que los pacientes deben adquirir habilidades prácticas para el cuidado del estoma en un corto periodo de tiempo, lo que a su vez puede originar problemas a nivel psicológico y un retraso en el desarrollo de la autonomía en el cuidado del estoma. 
El estudio de Araujo y Neves (2014) también tiene en cuenta a la familia como principal elemento en la adaptación al estoma, siendo la que se encarga de proporcionar apoyo emocional y social, con el fin de hacer frente a los problemas ante esta nueva situación y conseguir un adecuado autocuidado y autonomía del sujeto, tal y como lo corroboran diversos trabajos (Bonill y cols., 2013; Martín, y cols., 2010; Simmons, Smith, Bobb, y Liles, 2007).

Además, la literatura refiere que el personal de enfermería es esencial para que se lleve a cabo esta variable, ya que fomentan la cohesión familiar al favorecer un diálogo abierto donde puedan expresar sus preocupaciones y sentimientos. Sin embargo, en el artículo de Seng y cols. (2013), incluido en esta revisión, el elemento «apoyo familiar» no es identificado como variable que afecte al individuo.

A diferencia con el estudio de Araujo y Neves (2014), el estudio realizado por Seng y cols. (2013) tiene en cuenta la variable «diagnóstico del cáncer» como uno de los principales elementos que intervienen en la adaptación del usuario, siendo éste un generador de sentimientos de miedo y preocupación por la reaparición de la neoplasia tras la cirugía, o siendo vista dicha intervención como una oportunidad para sobrevivir.

Seng y cols. (2013) también refieren que tras el alta los pacientes continúan experimentando factores estresantes que dificultan la total aceptación de la enterostomía. Por eso, exponen que se debería proporcionar asesoramiento y apoyo a largo plazo y de forma continua.

A pesar de la gran cantidad de estudios relacionados con las ostomías, tras realizar una búsqueda exhaustiva, se procuraron encontrar las evidencias más actuales, por lo que se han recuperado únicamente dos revisiones sistemáticas, en las que se han podido observar resultados similares en cuanto a las variables que afectan al usuario en su adaptación. Sin embargo, con los estudios recuperados no se ha podido definir la importancia del estomaterapeuta en la adaptación del individuo a su enterostomía de eliminación.

Por otro lado, el presente trabajo de revisión presenta una serie de limitaciones. Una de ellas es la restricción por idioma, ya que al realizar la búsqueda únicamente en inglés y castellano se ha podido inducir un posible sesgo de publicación, ya que los documentos escritos en idioma inglés tienen más posibilidades de publicarse, recuperarse $y$, por tanto, citarse que los de otro idioma (Pértega y Pita, 2005).

Junto con el sesgo de publicación, el sesgo de selección representa una de las principales limitaciones metodológicas en este tipo de estudios, ya que es importante definir con precisión tanto los criterios de inclusión como los de exclusión de los estudios incluidos en la revisión, y que éstos sean lo más objetivos posibles (Pértega y Pita, 2005). Así pues, a pesar de que se definen con claridad los criterios de inclusión, los criterios de exclusión no están definidos. Esto es así, ya que al realizar la búsqueda únicamente con los criterios de elegibilidad, los resultados obtenidos ya eran excesivamente limitados, por lo que no se consideró conveniente restringir más la búsqueda estableciendo criterios de exclusión.

Otro aspecto que puede comprometer la validez de los resultados de esta revisión es la calidad de los estudios originales incluidos (Pértega y Pita, 2005), ya que a pesar de que los artículos incluidos tienen una calidad metodológica media y alta, al realizar el análisis para determinar dicho nivel de calidad, se utilizan diferentes checklist que en algunos casos son subjetivos y, por ende, dependen del criterio del autor, ya que la plataforma no cuenta con un sistema de validación posterior para corroborar los datos del registro (Servicio de Medicina Interna del Hospital de León, s.f.).

Otra de las limitaciones es el tamaño muestral de los estudios incluidos en las revisiones analizadas, sobre los que Seng y cols. (2013) se basan en su artículo, y del que Araujo y Neves (2014) no informan. Esta limitación puede deberse a la falta de registros de pacientes ostomizados. 
Teniendo en cuenta las deficiencias metodológicas observadas, se puede considerar que el elemento principal que repercute en la adaptación de los individuos es la educación sanitaria recibida, sobre todo, por parte del personal de enfermería.

A pesar de que algunos estudios recientes (Instituto Antae, 2014; Pittman, Kozell y Gray, 2015) refieren la minimización del impacto que origina la presencia del estoma en la calidad de vida de este tipo de pacientes y la reducción de costes sanitarios asociados, este estudio permite observar la carencia de investigaciones respecto al papel del especialista en estomaterapia.

Por ello es conveniente llevar a cabo nuevas investigaciones que contemplen las limitaciones de los distintos artículos publicados, sobre todo la ausencia de un registro de pacientes ostomizados que permita la realización de nuevos ensayos con un tamaño muestral adecuado, con el fin de obtener unas conclusiones con menor sesgo y mayor posibilidad de generalizar los resultados, así como evaluar la importancia del estomaterapeuta en el proceso de adaptación. La finalidad es dar a conocer al personal sanitario, la importancia de obtener unos conocimientos adecuados respecto a las variables que pueden afectar a este tipo de pacientes, encaminando los cuidados hacia la excelencia y, de este modo, disminuir el deterioro de la calidad de vida de los individuos.

\section{Referencias bibliográficas}

Aecc. (2014). Secuelas del cáncer de colon. Recuperado el 23 de febrero, 2015, de: https://www. aecc.es/sobreelcancer/cancerporlocalizacion/cancerdecolon/Paginas/secuelas.aspx.

Araujo Torquato Lopes, A. P. y Das Neves Decesario, M. (2014). The Adjustments Experienced by Persons With an Ostomy: An Integrative Review of the Literature. Ostomy wound management 60, 34-42.

Asociación Española de Cirujanos (2005). El Paciente Ostomizado en el entorno de una Asociación. Recuperado el 23 de febrero de 2015, de: http://www.aecirujanos.es/noticias/ paciente_ostomizado.php.

Bardin, L. (2002). Análisis de contenido ( $3^{\mathrm{a}}$ ed.). Madrid: AKAL.

Bonill de las Nieves, C., Hueso Montoro, C., Celdrán Mañas, M., Rivas Marín, C., Sánchez Crisol, I., y Morales Asencio, J. M. (2013). Viviendo con un estoma digestivo: la importancia del apoyo familiar. Index de Enfermería, 22, 209-213. doi:10.4321/S113212962013000300004.

Brown, H., y Randle, J. (2005). Living with a stoma: a review of the literature. Journal of Clinical Nursing, 14, 74-81. doi:10.1111/j.1365-2702.2004.00945.x.

Canaval G. E., Londoño M. E. y Herrera A. M. (2005). Guía de enfermería para el cuidado de la persona adulta con estoma. Recuperado el 24 de febrero, 2015, de: http://www.index-f. com/lascasas/documentos/lc0026.php.

Campillo, J. (2014). La calidad de vida de un paciente ostomizado es mayor al año de practicarse la cirugía que a los 6 meses. Recuperado el 3 de marzo de 2015, de: http://www. coloplast.es/ECompany/ESMed/Homepage.nsf/0/49b7e19ce680683bc1257ceb004bac9 8/\$FILE/Stoma Life.pdf.

Centro Cochrane Iberoamericano (2014). Revisiones Cochrane. Recuperado el 17 de marzo de 2015, de: http://es.cochrane.org/es/revisiones-cochrane.

Collado Boira, E. J. (2014). Autocuidados y calidad de vida en pacientes enterostomizados. Tesis doctoral. Universidad Cardenal Herrera, Valencia, España.

Corella Calatayud J. M., Vázquez Prado A., Tarragón Sayas M. A, Mas Vila T., Corella Mas J. M., Corella Mas L. (2005). Estomas, manual para enfermería. Alicante: CECOVA. Recuperado 
el 20 de febrero de 2015, de: http://www.bibliotecadigitalcecova.com/contenido/revistas/ cat6/pdf/libro_47.pdf.

Cotrim, H., y Pereira, G. (2008). Impact of colorectal cancer on patient and family: implications for care. European Journal of Oncology Nursing, 12, 217-26. doi:10.1016/j. ejon.2007.11.005.

Da Costa Santos M. C., Mattos Pimenta A. C., Cuce Nobre M. R. (2007). Estrategia pico para la construcción de la pregunta de investigación y la búsqueda de evidencias. Revista Latino-Americana de Enfermagem, 15, 508-511. doi:10.1590/S0104-11692007000300023.

Gómez del Río, N., Mesa Castro, N., Caraballo Castro, C., Fariña Rodríguez, A., Huertas Clemente, M. y Gutiérrez Fernández, Y. (2013). Los cuidados de enfermería en el impacto psicológico del paciente. ENE, 7(3). Recuperado el 25 de febrero de 2015, de: http://eneenfermeria.org/ojs/index.php/ENE/article/view/279.

Grupo CIDO (1997). La solución específica para cada necesidad. Draft. Recuperado el 10 de marzo de 2015, de: http://www.coloplast.es/ECompany/EsMed/homepage.nsf/0/e82b8e4 6e7d56ca241256a6a003e5ebb/\$FILE/Guia ostomizados.pdf.

Instituto Antae (2014). Coste-efectividad de la atención especializada en ostomía. Recuperado el 25 de febrero, 2015, de: http://www.enfermeriademurcia.org/attachments/ article/1753/141001. Dossier de prensa Estudio Coste Efectividad.pdf.

Letelier, L. M., Manríquez, J. J., y Rada, G. G. (2005). Revisiones sistemáticas y metaanálisis: ¿son la mejor evidencia? Revista Médica de Chile, 30, 37-39. doi:10.4067/S003498872005000200015.

López de Argumedo M., Reviriego E., Andrío E., Rico R., Sobradillo N. y Hurtado de Saracho I. (2006). Revisión externa y validación de instrumentos metodológicos para la lectura crítica y la síntesis de la evidencia científica. Informe de Evaluación de Tecnologías Sanitarias. Osteba núm. 2006/02.

Marín Martínez, F., Sánchez Meca, J., y López López, J. A. (2009). El metaanálisis en el ámbito de las Ciencias de la Salud: una metodología imprescindible para la eficiente acumulación del conocimiento. Fisioterapia, 31, 107-114. doi:10.1016/j.ft.2009.02.002.

Martín Muñoz, B., Panduro Jiménez, R. M., Crespillo Díaz, Y., Rojas Suárez, L., y González Navarro, S. (2010). El proceso de afrontamiento en personas recientemente ostomizadas. Index de Enfermería, 19, 115-119. doi:10.4321/S1132-12962010000200009.

Mejía Lopera, M. E. (2006). Reflexiones sobre la relación interpersonal enfermera-paciente en el ámbito del cuidado clínico. Index de Enfermería, 15, 48-52. Recuperado el 23 de febrero de 2015, de: http://scielo.isciii.es/scielo.php?script=sci_arttext\&pid=S1132$12962006000200010 \&$ Ing=es\&nrm=iso\&tlng=es.

Pértega Diaz, S., y Pita Fernández, S. (2005). Revisiones sistemáticas y Metaanálisis. Recuperado el 1 de abril de 2015, de: https://www.fisterra.com/mbe/investiga/metaanalisis/ RSyMetaanalisis.asp.

Pittman, J., Kozell, K., y Gray, M. (2015). Should woc nurses measure health-related quality of life in patients undergoing intestinal ostomy surgery?. Journal of Wound, Ostomy, and Continence Nursing, 36, 254-265. doi:10.1097/Won.0b013e3181a39347.

Seng Giap, M. A., Chen Chen, H., Chiew Siah, R. J., Hong-Gu, He, Klainin-Yobas, P. (2013). Stressors relating to patient psychological health following stoma surgery: an integrated literature review. Oncology Nursing Forum, 40, 587-94.

Servicio de Medicina Interna del Hospital de León (s. f). Fichas de lectura crítica FLC 2.0. Recuperado el 7 de abril de 2015, de: https://mileon.wordpress.com/2015/03/16/fichas-delectura-critica-flc-2-0/. 
Simmons, K. L., Smith, J. A., Bobb, K. A., y Liles, L. L. M. (2007). Adjustment to colostomy: Stoma acceptance, stoma care self-efficacy and interpersonal relationships. Journal of Advanced Nursing, 60, 627-635. doi:10.1111/j.1365-2648.2007.04446.x.

The Joanna Briggs Institute (2011). Joanna Briggs Institute Reviewer's Manual: 2011 Edition. Recuperado el 6 de abril de 2015, de: http://joannabriggs.org/assets/docs/sumari/ reviewersmanual-2011.pdf. 